\title{
Visualisation of contrast-filled stab wounds in various tissue types with computed tomography
}

\author{
Peter Mygind Leth*, Uffe Stolborg
}

Institute of Forensic Medicine, University of Southern Denmark, J.B. Winsløws Vej 17, DK-5000 Odense *pleth@health.sdu.dk

\begin{abstract}
Background: Stab wounds are common in homicide cases. Post-mortem multislice computed tomography (PMCT) has proved to be a useful tool in forensic examinations of victims of sharp force trauma, but due the limited resolution of soft tissues, the radiological depiction of a stab channel is difficult. In this study, we have tried to obtain information about the shape of a knife blade by CT scanning contrast-filled experimentally inflicted stab wounds in various types of pig tissue.

Methodology: The tissue samples were mounted on floral foam (oasis) with wooden sticks. Two contrast media were used: one was unmodified and easy flowing, and one was made more viscous with polyethylene glycol. Stab channels in ballistic soap were used for comparison. India ink-filled stab channels were investigated histologically to determine the pattern of leakage.

Principal findings: We found that the shape of the stab wounds on the CT images from lung and muscle tissue did not correspond well to the shape of the inflicting knife. There was a better correspondence in the images obtained from liver, spleen and kidney. The viscous contrast medium was less likely than the thin (easy flowing) contrast medium to spill into to structures outside the stab channel, but some spillage was observed for both types of contrast medium. Air bubbles were only observed in the viscous contrast medium.

Conclusion: Radiological evaluation of a contrast-filled stab wound in isolated tissue blocks did not permit the positive identification of the inflicting weapon, but it was, in tissue blocks from liver, spleen and kidney, possible to obtain a rough idea of the shape of the inflicting knife and to differentiate a knife from a screwdriver.
\end{abstract}

Keywords:

Stab wound, PMCT, Computed tomography, Forensic radiology, X-ray contrast

\section{INTRODUCTION}

This study addresses the use of contrast medium for the radiological visualisation of stab wounds. Stabbing is the most common homicide method in Denmark [1] and many other countries. The forensic investigation of stabbing cases is of great importance for police investigations and for legal assessments. Any procedure that could improve the quality of the forensic investigation would therefore be of interest. Post-mortem multislice computed tomography (PMCT) has proved to be a useful tool in forensic examinations, including in victims of sharp trauma [2]. In combination with post-mortem whole-body angiography [3-5], the vascular bed and vascular lesions can be depicted. However, due the limited resolution of soft tissues in PMCT, the radiological depiction of a stab channel is difficult. In cases of stabbing, it is important to identify the inflicting weapon. In this study, we have tried to obtain information about the shape of the knife blade by CT-scanning contrast-filled experimentally inflicted stab wounds in various types of pig tissue.

\section{MATERIALS AND METHODS}

Five single-edged knives with different shapes, including one with a serrated blade, and one screwdriver were used for this study (Table 1). The following types of pig tissue were used: liver, lung, muscle (m. psoas major), kidney and spleen. The tissue cubes (measuring approximately $5 \times 3 \times 7 \mathrm{~cm}$ long, broad and deep) were kept in a fixed position with floral foam (oasis) and wooden sticks. Two blocks of each tissue type were stabbed to a depth of $4 \mathrm{~cm}$ with each knife (60 tissue blocks in total). Visipaque (iodixanol $270 \mathrm{mg} / \mathrm{ml}$ ) was used as contrast medium, diluted to a radio opacity of 600 Hounsfield Units. The first of the stab wound channels for each knife/tissue type was scanned without contrast medium and then filled with unmodified contrast medium (thin contrast medium), the second was filled with viscous contrast medium produced by adding polyethylene glycol $(50 \% \mathrm{w} / \mathrm{w})$ to the thin contrast medium (viscous contrast medium). The contrast medium was instilled into the stab channel with a syringe until the channel was completely filled. Stab channels were also made in ballistic soap and filled with contrast medium. CT scanning was performed immediately after contrast instillation on a Siemens Somatom Spirit dual slice CT-scanner with a slice thickness of $1 \mathrm{~mm}$ and a reconstruction interval of $0.7 \mathrm{~mm}$. A mediumhard reconstruction algorithm was used (Siemens B70s). Two- and threedimensional reconstructions were generated. The images were viewed on a Leonardo workstation (syngo CT software, Siemens Medical Solutions).

Images of the stab channels from each sharp instrument obtained from the ballistic soap were superimposed in Photoshop on the stab channel images obtained from the various tissue blocks to evaluate whether the knife could be positively identified. Ballistic soap preserves a permanent imprint of the stab channel and served as gold standard for the shape and 
volume of the stab channels. The volume of the lower $3 \mathrm{~cm}$ of the stab channels and measurements of the thickness and width of the stab channels in the tissue blocks and in ballistic soap was calculated using the syngo CT software. The volume of air in the stab channels and the volume of contrast medium outside the stab channels were also measured. A new stab channel in each tissue type was filled with a suspension of India ink in warm water with gelatin, which solidifies when cooled. Longitudinal and transverse sections of the filled stab channels were obtained for microscopy to visualise how the contrast medium spread to structures outside the stab channel.

\section{RESULTS}

The results of the measurements are shown in Table 2. Stab channels without contrast medium were difficult to discern on the CT image, but parts of the stab channel could be observed in some cases. The volume of contrast required to fill the stab channel in lung tissue was higher than that in other types of tissue. This was most likely caused by the elasticity of the lung tissue, which caused the stab channels to become broadened, irregular and sometimes drop shaped, with contrast medium collecting in the bottom of the channel (Figures 1 and 2). It was somewhat difficult to distinguish between the contrast medium in the lung tissue and that in the stab channel due to seepage of medium into the surrounding lung tissue. In muscle tissue (psoas major), the stab channels were broad and deformed. In the liver, kidney and spleen, the CT images of the contrast-filled stab channels retained the shape of the inflicting knife to a greater extent than was observed in the lung or muscle tissue, but the channels were nevertheless still somewhat distorted (Figure 3).

There was always some spillage of contrast medium into the surrounding tissue, especially if a large hollow structure, such as the calyx system in the kidneys, was struck. A greater volume of contrast medium was observed outside the stab channel when thin contrast medium was used compared to viscous contrast medium containing polyethylene glycol (Table 2), but spillage was observed for both types of contrast. Stab channels filled with viscous contrast contained more air than did stab channels filled with thin contrast. Air bubbles (Figure 4) were only observed in the viscous contrast medium.

Table 1. Sharp instruments used in this study. The measures are in $\mathrm{mm} 4 \mathrm{~cm}$ from the tip.

\begin{tabular}{|c|c|c|c|c|c|}
\hline & Knife 1 & Knife 2 & Knife 3 & Knife 4 & \\
\hline Shape & & & & & \\
\hline Width of blade & 29,0 & 18,2 & 15,7 & 18,8 & \\
\hline Thickness of blade & 1,75 & 1,10 & 1,15 & 28,0 & 1,30 \\
\hline
\end{tabular}

Table 2. Volumes of contrast inside the stab channel (VS), contrast outside the stab channel (VO) and air in the stab channel (VA), in mm3. The last two columns show the percent contrast outside stab channels and percent air in stab channels for all stab channels in one type of tissue and contrast. TC=thin contrast, $\mathrm{VC}=$ viscous contrast.

\begin{tabular}{|c|c|c|c|c|c|c|c|c|c|c|c|c|c|c|c|c|c|c|c|c|c|}
\hline & & \multicolumn{3}{|c|}{ Knife 1} & \multicolumn{3}{|c|}{ Knife 2} & \multicolumn{3}{|c|}{ Knife 3} & \multicolumn{3}{|c|}{ Knife 4} & \multicolumn{3}{|c|}{ Knife 5} & \multicolumn{3}{|c|}{ Skrewdriver } & All & All \\
\hline \multicolumn{2}{|c|}{ Shape } & & & \multicolumn{3}{|c|}{ 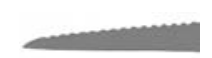 } & & \multicolumn{3}{|c|}{ 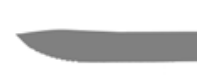 } & \multicolumn{3}{|c|}{$\square$} & & \\
\hline & & VS & vo & VA & VS & vo & VA & VS & vo & VA & VS & vo & VA & VS & vo & VA & VS & vo & VA & VO/vs & VA/VS \\
\hline \multirow{2}{*}{ Liver } & $\mathrm{TC}$ & 130 & 30 & 10 & 110 & 100 & 10 & 110 & 30 & 10 & 110 & 70 & 20 & 130 & 30 & 10 & 200 & 150 & 80 & $51 \%$ & $18 \%$ \\
\hline & VC & 170 & 20 & 30 & 110 & 0 & 20 & 140 & 10 & 20 & 120 & 30 & 20 & 120 & 40 & 20 & 410 & 280 & 190 & $36 \%$ & $28 \%$ \\
\hline \multirow{2}{*}{ Lung } & $\mathrm{TC}$ & 250 & 60 & 20 & 290 & 180 & 50 & 470 & 10 & 250 & 640 & 40 & 410 & 200 & 30 & 20 & 550 & 120 & 60 & $18 \%$ & $34 \%$ \\
\hline & VC & 650 & 10 & 80 & 760 & 50 & 110 & 540 & 80 & 80 & 420 & 20 & 110 & 310 & 40 & 10 & 600 & 90 & 240 & $9 \%$ & $19 \%$ \\
\hline \multirow{2}{*}{ Muscle } & TC & 840 & 0 & 220 & 590 & 520 & 10 & 190 & 120 & 50 & 810 & 170 & 30 & 280 & 20 & 20 & 390 & 40 & 10 & $28 \%$ & $11 \%$ \\
\hline & VC & 820 & 0 & 190 & 220 & 140 & 50 & 290 & 20 & 70 & 450 & 120 & 50 & 220 & 10 & 10 & 420 & 20 & 30 & $13 \%$ & $17 \%$ \\
\hline \multirow{2}{*}{ Kidney } & $\mathrm{TC}$ & 220 & 80 & 80 & 140 & 60 & 0 & 80 & 70 & 10 & 80 & 20 & 0 & 120 & 30 & 10 & 370 & 40 & 10 & $30 \%$ & $11 \%$ \\
\hline & VC & 190 & 10 & 0 & 110 & 0 & 30 & 90 & 10 & 20 & 100 & 10 & 30 & 120 & 10 & 20 & 400 & 30 & 30 & $7 \%$ & $13 \%$ \\
\hline \multirow{2}{*}{ Spleen } & $\mathrm{TC}$ & 130 & 170 & 10 & 100 & 100 & 10 & 120 & 20 & 20 & 120 & 40 & 10 & 90 & 10 & 10 & 420 & 30 & 30 & $38 \%$ & $9 \%$ \\
\hline & VC & 150 & 140 & 20 & 150 & 0 & 40 & 120 & 0 & 10 & 120 & 20 & 20 & 130 & 0 & 20 & 390 & 20 & 10 & $17 \%$ & $11 \%$ \\
\hline $\begin{array}{l}\text { Ballistic } \\
\text { soap }\end{array}$ & TC & 140 & 0 & 0 & 130 & 0 & 0 & 80 & 0 & 0 & 90 & 0 & 0 & 140 & 0 & 0 & 400 & 0 & 0 & & \\
\hline
\end{tabular}




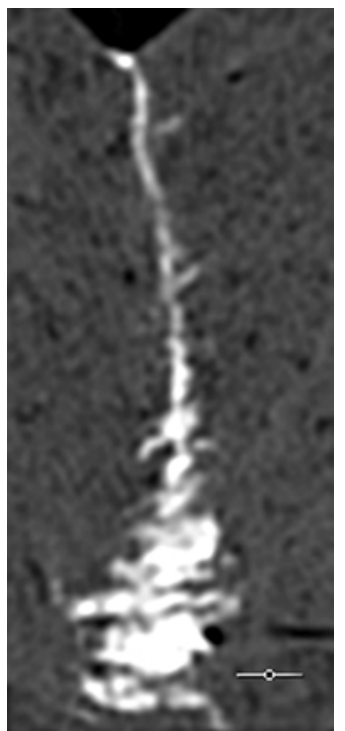

Figure 1. 2D section of a stab channel, knife 2, lung tissue, thin contrast medium. The scale bar is $5 \mathrm{~mm}$.

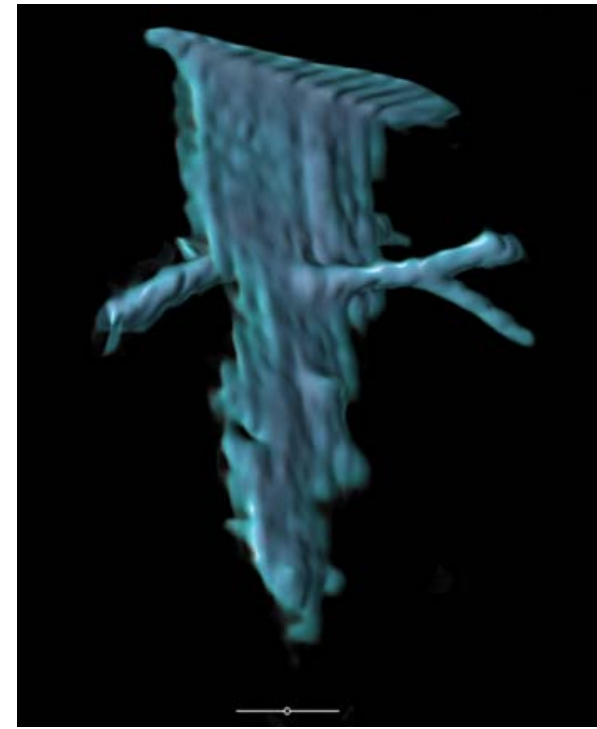

Figure 3. 3D reconstruction of a stab channel, knife 2 , liver, viscous contrast medium. The scale bar is $5 \mathrm{~mm}$.

Microscopy of the India ink-filled stab channels showed leakage into blood vessels, bile ducts, bronchi, renal tubules and calyces (Figure 5).

The stab channels in ballistic soap were slightly smaller than the knife blades due to elasticity of the soap. It was not possible to positively identify a knife by superimposition in Photoshop, but in liver, kidney and spleen samples, it was possible to rule out a specific knife if the difference between the blades was large, and it was easy to distinguish the image of a contrast-filled stab channel produced by a screwdriver from that produced by a knife. Knife 3 was serrated, but this could not be established by the $\mathrm{CT}$ images.

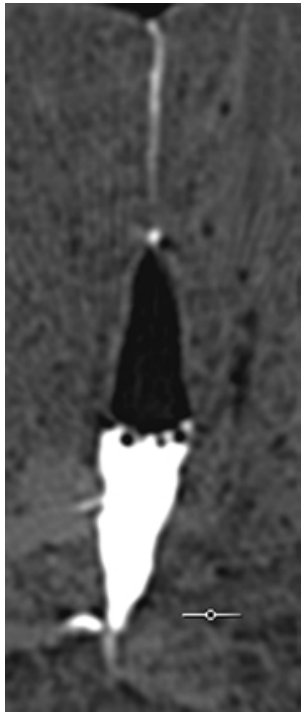

Figure 2. 2D section of a stab channel, knife 1, lung tissue, viscous contrast medium. The scale bar is $5 \mathrm{~mm}$.

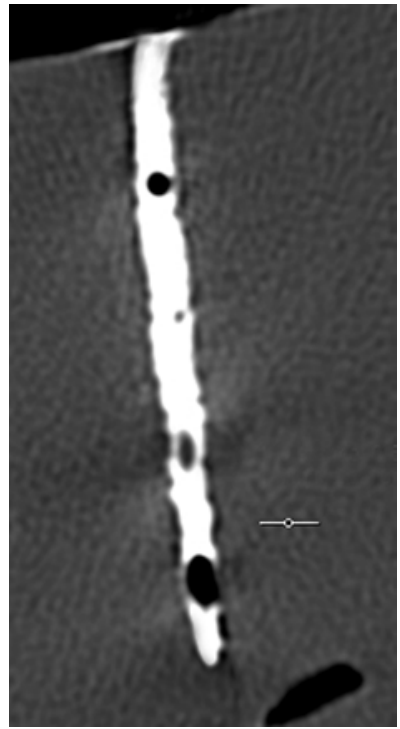

Figure 4. Bubbles in viscous contrast medium, 2D section of a stab channel, liver, screwdriver. The scale bar is $5 \mathrm{~mm}$.

\section{DISCUSSION}

In this study, we have developed and tested a method for the radiological evaluation of contrast-filled stab wounds in various types of tissue. The shape of a stab wound is to some degree dependent on the inflicting knife, and it is often important to determine which knife has caused the wound or at least which type of sharp weapon was used. It has previously been shown that it is possible to create a virtual cast of a stab wound in cartilage using micro-computed tomography, although it remains to be seen whether this method has any advantages compared to traditional casts made using dental 


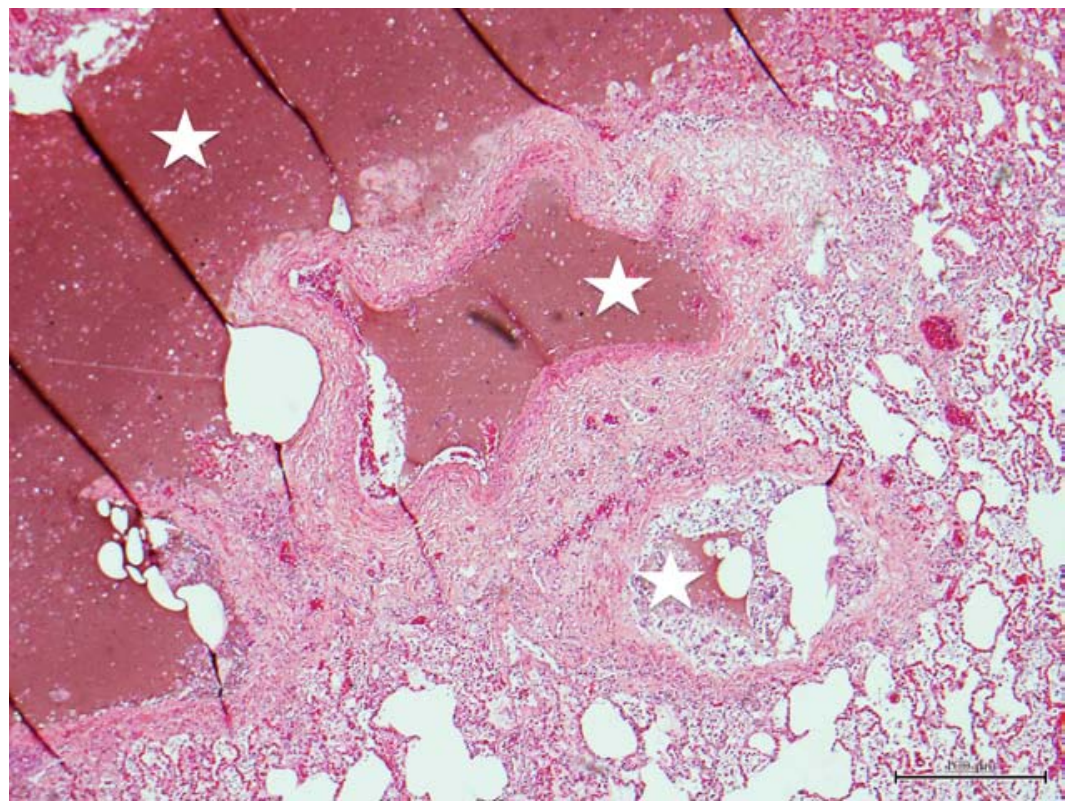

Figure 5. Stab channel in lung tissue filled with India ink, demonstrating the spread of the India ink to an artery and a bronchus. The India ink is marked with asterisks.

impression material [6]. The estimation of stab wound direction and depth in contrast-filled experimentally inflicted stab wounds in pig muscle tissue has also been tried [7]. Many of the stab wounds that we encounter in our forensic practice involve thoracic or abdominal organs or muscles such as the gluteus maximus and quadriceps femoris [8]. Some stab wounds can be detected by conventional post mortem $\mathrm{CT}[2,9,10]$, and vascular injuries can be evaluated with post mortem whole-body angiography [11]. A radiological depiction of the stab channels that permits the characterisation of the inflicting weapon is not possible with these methods. The general wound morphology may be obtained by dissection, but this is not a very precise method, and it destroys the evidence in the process. Because computerised tomography is used routinely in our department [12], it was reasonable to attempt to obtain radiological images of stab channels after filling them with contrast medium. We experimented with different mounting methods and found that mounting the tissue samples with floral foam (oasis) and wooden sticks was the best way to keep the samples in position. It should be kept in mind that the mounting procedure may cause some distortion of the wound track. There were several difficulties in obtaining useful images of the wound track. In lung tissue, the contrast medium enters the porous lung tissue, even when a viscous contrast medium is used, and it becomes somewhat difficult to distinguish between the stab channel and the surrounding tissue. The measurements of contrast outside the stab channels in lung tissue, given in Table 2, show that although a large volume of contrast was present outside of the stab channel, the volume of contrast in the stab channel in lung tissue is also relatively high. The latter is most likely caused by the elasticity of the lung tissue, which causes the stab channel to become broadened, irregular and sometimes drop shaped. Muscle tissue, in this case psoas major, presented other problems. The muscle was soft and pasty and could be moulded, almost like plasticine. The shape of the stab wounds on the $\mathrm{CT}$ images therefore did not correspond to the shape of the inflicting knife. It is quite possible that a stab wound inflicted to a muscle in vivo would behave differently. Liver, spleen and kidney tissue yielded better results, but it was difficult to prevent the stab channel from becoming distorted, and there was always some spillage of contrast medium. The viscous contrast medium was slightly less prone to seeping out into the surrounding tissue than the thin contrast medium. The leakage could readily be distinguished from the contrast medium in the stab channels, and its volume was easily measured, so the leakage did not in itself present a major problem. The exception was in lung tissue, in which it was sometimes difficult to find the border between the stab channel and the surrounding tissue. Sometime the viscous contrast medium contained air bubbles, but this did not distort the image. However, this study does not indicate that the use of viscous contrast medium presents a great advantage. The $3 \mathrm{DM} C \mathrm{CT}$ reconstructions of the stab channels in tissue were was not shaped exactly as the corresponding stab channels in ballistic soap, and it was not possible to obtain a precise identification of the inflicting knife, but it was usually possible to get a crude impression of the shape of knife blade and to make a distinction between a knife and a screwdriver. However, it must be remembered that stab wounds in real life are different from the experimentally inflicted "stab wounds" in this study. The knife may be moved in the stab channel relative to the body and/or withdrawn at a different angle; the knife could also enter the wound several times, making the stab channel much more irregular.

\section{CONCLUSION}

Radiological analysis of a contrast-filled stab wound in isolated tissue blocks did not permit the positive identification of the inflicting sharp weapon, but it was, in tissue blocks from liver, spleen and kidney, possible to determine the general shape of the inflicting knife and to differentiate a knife from a screwdriver. 


\section{REFERENCES}

[1] Leth PM. Homicides in Southern Denmark. Homicide Studies. 2010;14(4):419-35.

[2] Schnider J, Thali MJ, Ross S, Oesterhelweg L, Spendlove D, Bolliger SA. Injuries due to sharp trauma detected by post-mortem multislice computed tomography (MSCT): a feasibility study. Leg Med (Tokyo). 2009 Jan;11(1):4-9.

[3] Ross S, Spendlove D, Bolliger S, Christe A, Oesterhelweg L, Grabherr S, et al. Postmortem whole-body CT angiography: evaluation of two contrast media solutions. AJR Am J Roentgenol. 2008 May;190(5):1380-9.

[4] Jackowski C, Persson A, Thali MJ. Whole body postmortem angiography with a high viscosity contrast agent solution using poly ethylene glycol as contrast agent dissolver. J Forensic Sci. 2008 Mar;53(2):465-8.

[5] Ehrlich E, Farr T, Maxeiner H. Detection of arterial bleeding points in basilar subarachnoid hemorrhage by postmortem angiography. Leg Med (Tokyo). 2008 Jul;10(4):171-6.

[6] Pounder DJ, Sim LJ. Virtual Casting of Stab Wounds in Cartilage Using Micro Computed Tomography. Am J Foren Med Path. 2011 Jun;32(2):97-9.
[7] Bolliger SA, Preiss U, Glaeser N, Thali MJ, Ross S. Radiological stab wound channel depiction with instillation of contrast medium. Legal Medicine. 2010;12(1):39-41.

[8] Leth PM. Lethal Stabbings in Southern Denmark. The Scandinavian Journal of Criminal Law and Criminology 2008;3:279-86.

[9] Leth PM, Christensen MR. Computerized tomography used for investigation of homicide victims. Scandinavian Journal of Forensic Science. 2011;17(1):11-6.

[10] Schnider J Fau - Thali MJ, Thali Mj Fau - Ross S, Ross S Fau - Oesterhelweg L, Oesterhelweg L Fau - Spendlove D, Spendlove D Fau - Bolliger SA, SA B. - Injuries due to sharp trauma detected by post-mortem multislice computed tomography (MSCT): a feasibility study. Leg Med. 2009;11(1):4-9.

[11] Grabherr S, Djonov V, Yen K, Thali MJ, Dirnhofer R. - Postmortem angiography: review of former and current methods. AJR Am J Roentgenol. 2007;188(3):832-8.

[12] Leth PM. Computerized tomography used as a routine procedure at postmortem investigations. Am J Forensic Med Pathol. 2009 Sep;30(3):219-22. 